\title{
ON THE THERMAL INTERPRETATION OF HARD X-RAY BURSTS FROM SOLAR FLARES
}

\author{
JOHN C. BROWN \\ Dept. of Astronomy, University of Glasgow, Glasgow, Scotland
}

\begin{abstract}
The possible validity of thermal bremsstrahlung models of flare hard X-ray bursts is investigated quantitatively. In particular, the problem of rapid thermal conduction in 'multi-temperature' models is adequately examined for the first time by using a continuous temperature distribution consistent with the observed X-ray spectrum. This distribution is obtained from a general analytic solution for the temperature structure required to mimick any 'non-thermal' spectrum, the method being equally applicable to cosmic sources.

It is concluded that the thermal interpretation might extend to $\mathrm{X}$-rays of hundreds of $\mathrm{keV}$, a result with important consequences for flare energetics. The relationship of such a model to observations of $\mathrm{X}$-ray polarization and rapid time variations is also considered.
\end{abstract}

\section{Introduction}

There is a general consensus in the literature that flare X-ray emission is dominated at low energies by thermal bremsstrahlung, as evidenced by continuum and line spectra (e.g. Neupert, 1969), and at high energies by non-thermal emission from the energetic particles observed directly in space (e.g. Fichtel and McDonald, 1967) and by means of their radio (Takakura, 1967) and $\gamma$-ray emissions (Chupp et al., 1973). However, there is still considerable uncertainty as to the photon energies at which the transition occurs between these two types of emission. The general trend is to accept that thermal bremsstrahlung does not extend to more than a few tens of $\mathrm{keV}$, and probably less than $10 \mathrm{keV}$, emission harder than this arising by bremsstrahlung of non-thermal electron streams (e.g. De Jager, 1967). Chubb (1971) has, however, recently reiterated the possibility of a multi-temperature thermal source, previously advocated by Chubb et al. (1966), which might fit the observations up to hundreds of $\mathrm{keV}$.

Resolution of this question is crucial to considerations of flare models, particularly in respect of energy distribution in the flare, since the steep spectrum of non-thermal flare particles implies that the bulk of their energy resides at the low energy end of their distribution (e.g. Neupert, 1968; Brown, 1971). If thermal X-ray emission is dominant only up to ten $\mathrm{keV}$ or so, the non-thermal electron spectrum must extend down to this energy implying a large total energy amongst these particles, comparable with the total flare energy release in fact (Brown, 1971, 1972a, 1973a). This situation bears heavily on particle acceleration mechanisms, requiring that much of the magnetic energy released go directly into particles, and permitting the possibility that it is these which subsequently heat the thermal flare or at least part of it (e.g. Neupert, 1968; Kane and Donnelly, 1971; Brown, 1973b). Alternatively, if thermal $\mathrm{X}$-rays dominate the spectrum to around $100 \mathrm{keV}$, the energetic electrons contributing the high energy tail must carry only a relatively small part of the flare energy, 
particle acceleration being then only a minor artefact of the magnetic energy release.

The tendency for most authors to accept the dominance of non-thermal electron bremsstrahlung above about $10 \mathrm{keV}$ can be attributed to a number of objections raised against the multi-temperature model proposed by Chubb(1971). Firstly, there are the observations of polarization (Tindo et al., 1972) and rapid time variation (e.g. Frost, 1969), in the hard X-ray emission, thought to be uncharacteristic of thermal emission (Tindo et al., 1970; Kahler, 1971a). Secondly, the high temperatures $\left(\gtrsim 10^{8} \mathrm{~K}\right)$ needed in a multi-temperature model to produce the observed hard X-ray spectrum imply the existence of steep temperature gradients in the source and consequent rapid energy redistribution by thermal conduction, by electron escape, and by radiation (Culhane et al., 1970). On these grounds, Kahler (1971a, b) calculates that the multitemperature source would not survive long enough to sustain the observed hard X-ray burst.

It is the aim of this paper to demonstrate that these objections have not yet been adequately formulated physically and that when the formulation is complete, a multitemperature source is not at present precluded from explaining currently available $\mathrm{X}$-ray data to over $100 \mathrm{keV}$. In particular it is pointed out that conduction in the multi-temperature source can only be correctly described on the basis of a temperature structure determined by the requirement of fitting the observed hard X-ray spectrum and not by the simple empirical models of the temperature structure used by Kahler (1971a) (cf. Culhane et al., 1970). Such a self-consistent description of the multi-temperature X-ray flare model is developed here from a general analytic solution for the temperature structure required to mimic any non-thermal X-ray spectrum. This solution may also be of interest in the field of cosmic X-ray source models, some of which involve a multi-temperature formulation (e.g. Greisen, 1971; Sunyaev and Illarionov, 1972). In the last section of the paper, possibilities of explaining also the observed hard X-ray polarization and time variations in terms of the multitemperature model are considered.

\section{X-Ray Continuum Spectra from Distributed Temperature Sources}

For a thermal bremsstrahlung source at distance $R$, the continuum photon energy flux at the Earth is

$$
F(\varepsilon)=\frac{8.1 \times 10^{-39}}{R^{2}} \int_{V} \frac{e^{-\varepsilon / k T}}{T^{1 / 2}} n^{2} \mathrm{~d} V\left(\mathrm{keV} \mathrm{cm}^{-2} \mathrm{~s}^{-1} \mathrm{keV}^{-1}\right),
$$

where $n(\mathbf{r}), T(\mathbf{r})$ are the electron density $\left(\mathrm{cm}^{-3}\right)$ and temperature at position $\mathbf{r}$ in the source volume $V$ and $\varepsilon$ is the photon energy (e.g. Culhane, 1969), variation of the Gaunt factor from unity being neglected. The simplest treatment of the non-isothermal model to fit photon spectra which deviate from the isothermal (exponential) form is merely by trial summing of several contributions of isothermal type, each with its temperature $T$ and emission measure $n^{2} \Delta V$ as adjustable parameters - i.e. the inte- 
gral (1) is approximated by a few term sum (e.g. Cline et al., 1969; Herring and Craig, 1973; Batestone et al., 1970).

A more sophisticated approach is to insert trial fitting functions for $n(\mathbf{r})$ and/or $T(\mathbf{r})$ into (1) until the required $F(\varepsilon)$ is mimicked (similarly to the fitting of trial powerlaw spectra in the case of non-thermal bremsstrahlung e.g. Kane and Anderson, 1970). Milkey (1971) has briefly considered this approach in connection with flares while Chambe (1971) applied it to soft X-rays from coronal condensations. Illarionov and Sunyaev (1972) (see also Felten and Rees, 1972) have investigated the case of powerlaw forms for $n, T$ as functions of depth in a hot radiating atmosphere and shown how this can produce a power law for $F(\varepsilon)$. Their investigation is primarily concerned with cosmic sources but also mentions possible relevance of this result to the characteristic power law of solar flare hard X-rays.

As will now be demonstrated, however, the problem of fitting an arbitrary observed X-ray spectrum $F(\varepsilon)$ by a distributed temperature source can in fact be solved in an entirely general way.

Let the source volume $V$ be divided into elements $\mathrm{d} V$ such that $\mathrm{d} V$ contains all the plasma for which the electron temperature lies in the interval $T, \mathrm{~d} T$. It is then natural to define an emission measure $\mu(T)$ per unit temperature $\left(\mathrm{cm}^{-3} \mathrm{~K}^{-1}\right)$ such that

$$
\mu(T) \mathrm{d} T=n^{2} \mathrm{~d} V .
$$

The significance of this definition is straightforward so long as a unique value of $n$ may be associated with each $T$, as will be the case in a stratified source (see Section 3 ). If this condition is not met, $V$ may be subdivided into elements in each of which it is valid and the definition of $\mu(T)$ modified by replacing the right side of Equation (2) by a discrete sum over these elements. For the present purpose it will be convenient to express $T$ and $\mu$ in more appropriate units, viz.

and

$$
T=10^{6} T_{6}
$$

$$
\mu(T) \mathrm{d} T=10^{41} \mu^{*}\left(T_{6}\right) \mathrm{d} T_{6}
$$

where $T_{6}$ is now in millions of ${ }^{\circ} \mathrm{K}$ and $\mu^{*}\left(T_{6}\right)$ is the emission measure per $10^{6} \mathrm{~K}$ in units of $10^{41} \mathrm{~cm}^{-3}$. Then (1) becomes

$$
F(\varepsilon)=\frac{0.81}{R^{2}} \int_{T_{6}=0}^{\infty} \frac{\mu^{*}\left(T_{6}\right)}{T_{6}^{1 / 2}} e^{-11.6 \varepsilon / T_{6}} \mathrm{~d} T_{6}
$$

where $\varepsilon$ is in $\mathrm{keV}$.

Changing the variable to the inverse temperature parameter

$$
t=11.6 / T_{6}
$$

and defining

$$
G(t)=\mu^{*}\left(T_{6}\right) T_{6}^{3 / 2}
$$


then Equation (4) becomes simply

$$
F(\varepsilon)=\frac{7 \times 10^{-2}}{R^{2}} \int_{t=0}^{\infty} G(t) e^{-\varepsilon t} \mathrm{~d} t
$$

This equation shows at once that the non-isothermal bremsstrahlung spectrum $F(\varepsilon)$ is essentially the Laplace Transform of the temperature distribution $G-$ i.e.

$$
F(\varepsilon)=\frac{7 \times 10^{-2}}{R^{2}} \mathscr{L}\{G(t) ; \varepsilon\}
$$

Thus, in an entirely general way, the temperature distribution required to mimic any observed 'non-thermal' spectrum (i.e. non-isothermal) is obtained immediately on simply inverting integral Equation (8), i.e.

$$
G(t)=14.3 R^{2} \mathscr{L}^{-1}\{F(\varepsilon) ; t\}
$$

which, on re-expression in the original variables, yields

$$
\mu^{*}\left(T_{6}\right)=\frac{14.3 R^{2}}{T_{6}^{3 / 2}} \mathscr{L}^{-1}\left\{F(\varepsilon) ; \frac{11.6}{T_{6}}\right\}
$$

Since the Inverse Transform $\mathscr{L}^{-1}$ may be obtained for a very wide range of functions (e.g. Sneddon, 1972) this clearly shows the inadequacy of spectral data alone for establishing the existence of non-thermal processes in X-ray sources. Two particular examples of spectra may be used as illustrations. Firstly, the commonly used powerlaw fit to 'non-thermal' spectra, i.e. $F(\varepsilon)=A \varepsilon^{-\gamma+1}$ (where $\gamma$ is the photon number flux index), is mimicked by a thermal emission measure distribution given by

$$
\begin{aligned}
\mu^{*}\left(T_{6}\right) & =\frac{14.3 R^{2}}{T_{6}^{3 / 2}} \mathscr{L}^{-1}\left\{A \varepsilon^{-\gamma+1} ; \frac{11.6}{T_{6}}\right\}= \\
& =\frac{14.3 R^{2}}{T_{6}^{3 / 2}} A\left(\frac{11.6}{T_{6}}\right)^{\gamma-2} \frac{1}{\Gamma(\gamma-1)},
\end{aligned}
$$

where $\Gamma$ is the gamma function (e.g. Abramowitz and Segun, 1965), i.e.

$$
\mu^{*}\left(T_{6}\right)=\frac{0.36 A R^{2}}{\Gamma(\gamma-1)}\left(\frac{T_{6}}{11.6}\right)^{-\gamma+1 / 2}
$$

which is the same result as obtained by Illarionov and Sunyaev (1972) in a less general way. The form of $\mu^{*}$ given by (11) is illustrated in Figure 1 for the burst recorded by Cline et al. (1969) - see also Section 5.

A second example of some physical interest, which happens to have an analytic solution, arises in connection with flares which sometimes exhibit a power law spectrum with high energy cut-off (e.g. Cline et al., 1968; Frost, 1969) above some energy $\varepsilon_{1}$ (of order $100 \mathrm{keV}$ ). This may be approximately represented as $F(\varepsilon)=$ 


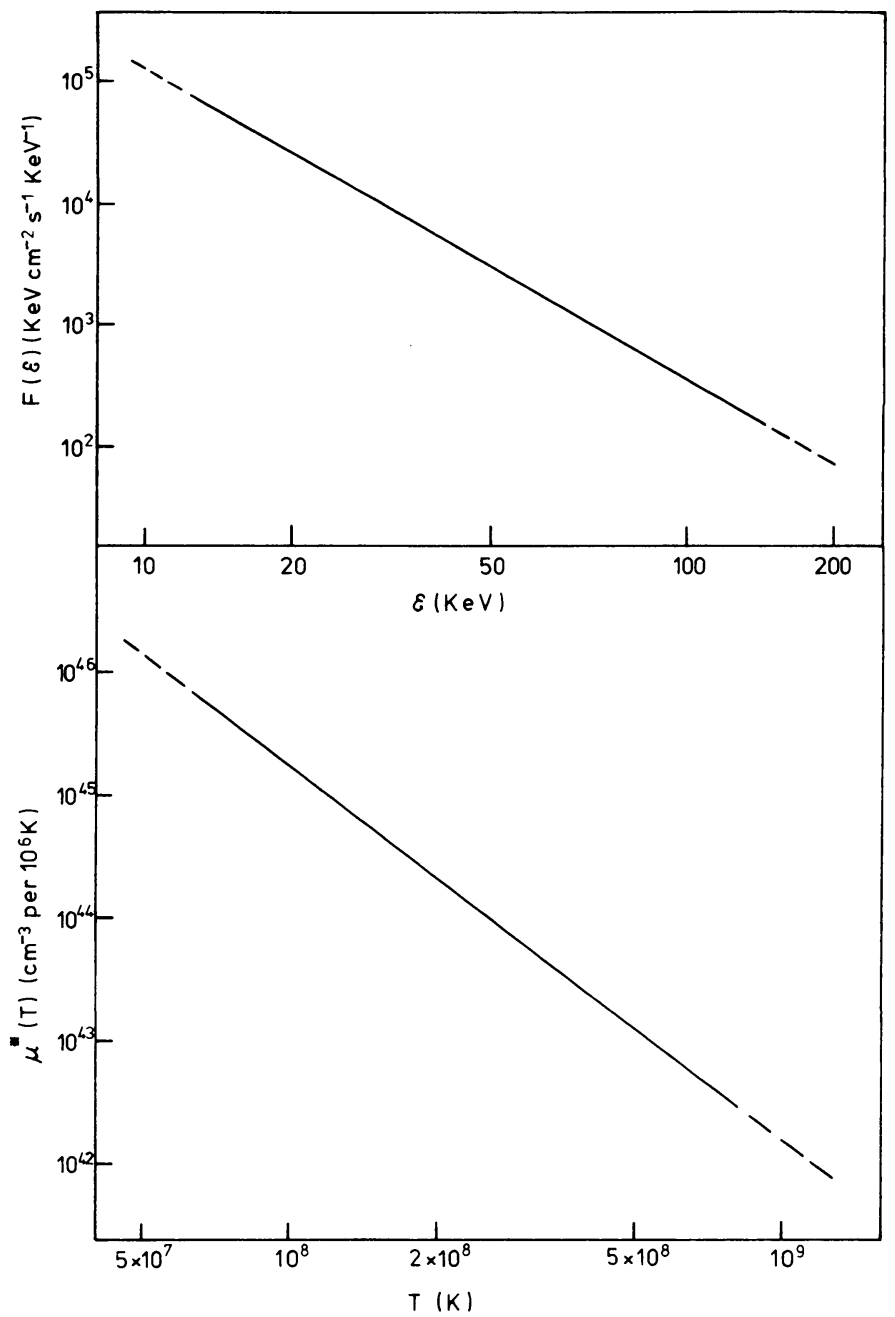

Fig. 1. Photon spectrum $I(\varepsilon)$ for a hard X-ray burst with $\gamma=3.5$ and a peak flux $I_{0}=300$ photons $\mathrm{cm}^{-2}$ $\mathrm{s}^{-1}$ above $80 \mathrm{keV}$ (Cline et al., 1969), together with the distribution of emission $\mu(T)$ required to produce it thermally.

$=A \varepsilon^{-\gamma+1} e^{-\sqrt{\varepsilon / \varepsilon_{1}}}$ (cf. Elwert and Haug, 1971). The temperature structure required to fit this is then, by (10),

$$
\begin{aligned}
\mu^{*}\left(T_{6}\right) & =\frac{14.3 R^{2}}{T_{6}^{3 / 2}} A \mathscr{L}^{-1}\left\{\varepsilon^{-\gamma+1} e^{-\sqrt{\varepsilon / \varepsilon_{1}}} ; \frac{11.6}{T_{6}}\right\}= \\
& =3.7 \times 10^{-2} A R^{2}\left(\frac{T_{6}}{46.4}\right)^{-\gamma+1 / 2} i^{(2 \gamma-4)} \operatorname{erfc}\left(\sqrt{\frac{T_{6}}{46.4 \varepsilon_{1}}}\right),
\end{aligned}
$$

where $i^{n}$ erfc $x$ is the $n$th repeated integral of the error function (Ambramowitz and Segun, 1965). 
Spectra which, unlike the above, cannot be readily fitted by analytic forms or for which the Inverse Transform is not expressible in terms of common analytic functions may nevertheless be treated by numerical transform methods (see e.g. Sneddon, 1972). The only factor not included in the above analysis is the Gaunt factor. Its inclusion would, however, add only a weighting function to $\mu(T)$.

It may be apt to emphasize that solution (10) is only meaningfully applicable to the high temperature range of $\mu(T)$ since the assumptions involved in Equation (1) refer only to hard photon energies (i.e. lines are omitted, etc.). This is clearly so since, for example, distribution (11) implies infinite amounts of plasma at zero temperature because the photon spectrum $A \varepsilon^{-\gamma+1}$ has been formally extrapolated to small $\varepsilon$ in the integrand. In fact, however, such formal extrapolation will cause little error in solution (11) since, on the one hand, material of $k T \ll \varepsilon$ cannot significantly contribute to $F(\varepsilon)$ due to the exponential in (1) while, on the other, the steepness of typical spectra $F(\varepsilon)$ (and hence $\mu(T)$ ) ensures that low energy photons emanate predominantly from low temperature material. That is, as is already known, X-ray photons of energy $\varepsilon$ can be quite closely identified with material at $k T \simeq \varepsilon$, so that solution (10) at temperature $T$ has an accuracy comparable with that of $F(\varepsilon=k T)$. To be completely general, of course, (10) should have been solved for the entire observed spectrum $F(\varepsilon)$ - i.e. a power-law merging with an exponential at energies around $1-20 \mathrm{keV}$, corresponding approximately to a power-law temperature structure $\mu(T)$ of type (11) at high $T$ merging into an isothermal region ( $\mu a \delta$-function) at a few tens of millions ${ }^{\circ} \mathrm{K}$. For the higher temperature regime investigated here, however, this refinement will not be necessary.

The most general use of solution (10) must lie in the accurate inference of the temperature distribution in sources (cosmic and solar) believed, from other considerations, to be of thermal type. Section 3 of this paper relates to the interpretation of $\mu(T)$ deduced in this way in the construction of models of such sources. In cases where the non-isothermal interpretation is in doubt, as it is in flares, recourse must be had to physical, and particularly energetic, considerations to assess the true nature of the source by eliminating either the thermal or non-thermal alternatives c.f. Kahler (1971a). Solution (10) provides accurately the temperature structure, demanded by the $\mathrm{X}$-ray spectral data, required for such investigations. In Section 4, this method is applied to the problem of cooling of hot multi-temperature plasmas in flares.

\section{Physical Data on Sources from the Differential Emission Measure}

To be useful beyond the immediate context of interpreting the $\mathrm{X}$-ray spectrum, the differential emission measure function $\mu^{*}\left(T_{6}\right)$ obtained in Section 2 must be more explicitly expressed in terms of the source structure. In particular, the variables $n$ and $T_{6}$ must be separately extracted from their combination in $\mu^{*}$. In the presence of the typically large temperature gradients resulting from the solution of (10) (e.g. (11)), approximately constant pressure is likely to prevail through the source (cf. the transition region in the quiet Sun) unless a highly randomised magnetic field isolates 
individual plasma elements. In their cooling calculations for high temperature coronal flare plasmas, both Culhane et al. (1970) and Kahler (1971a, b) have characterized the source by a single (constant) density - a state of affairs which is highly unlikely due to the strong pressure gradients resulting from it. This distinction is important since variations in $\boldsymbol{n}$ modify the temperature gradient deduced from $\mu^{*}\left(T_{6}\right)$ (see below) and so affect the inferred conductive flux (see Section 4). For the remainder of this paper, therefore, it will be supposed that constant pressure prevails in the source so that

$$
n(\mathbf{r}) T_{6}(\mathbf{r})=10 \times n_{10},
$$

where $n_{10}$ is the density $\left(\mathrm{cm}^{-3}\right)$ where the temperature is $10^{7} \mathrm{~K}$ (i.e. $\left.T_{6}=10\right)$ - roughly where the source becomes near isothermal as determined from soft X-ray data (e.g. Neupert, 1969).

Relation (13), together with (2) immediately yields the volume $\mathrm{d} V$ of plasma in a given temperature range $\mathrm{d} T_{6}$, viz.

$$
\mathrm{d} V\left(\mathrm{~cm}^{3}\right)=10^{39} \frac{\mu^{*}\left(T_{6}\right)}{n_{10}^{2}} T_{6}^{2} \mathrm{~d} T_{6}
$$

so that the total volume $\Delta V\left(T_{1}, T_{2}\right)$ lying between temperatures $T_{1}$ and $T_{2}$

$$
\Delta V\left(T_{1}, T_{2}\right)=\frac{10^{39}}{n_{10}^{2}} \int_{T_{1}}^{T_{2}} \mu^{*}\left(T_{6}\right) T_{6}^{2} \mathrm{~d} T_{6} .
$$

If a one-dimensional model is adopted for the source, with $n$ and $T$ varying along the $Z$ coordinate, and with cross sectional area $\Sigma$, then (14) and (15) can be written

$$
\mathrm{d} Z(\mathrm{~cm})=10^{39} \frac{\mu^{*}\left(T_{6}\right) T_{6}^{2}}{n_{10}^{2} \Sigma} \mathrm{d} T_{6}
$$

and

$$
\Delta Z\left(T_{1}, T_{2}\right)=\frac{10^{39}}{n_{10}^{2} \Sigma} \int_{T_{1}}^{T_{2}} \mu^{*}\left(T_{6}\right) T_{6}^{2} \mathrm{~d} T_{6},
$$

where $\mathrm{d} Z$ is the 'length' of the source lying in temperature interval $\mathrm{d} T_{6}$ and $\Delta Z\left(T_{1}, T_{2}\right)$ is the 'length' between $T_{6}=T_{1}$ and $T_{6}=T_{2}$.

This one-dimensional geometry is appropriate to the hot stratified atmosphere of some cosmic X-ray source models (e.g. Illarionov and Sunyaev, 1972) and also describes sources of filamentary type comprising trapped plasma in a uniform magnetic flux tube such as appears to occur in the solar corona (e.g. Culhane et al., 1970). In this latter case, $Z$ is measured along the field.

Equation (16) additionally defines the temperature gradient at each point in the 
source, viz.

$$
\frac{\mathrm{d} T_{6}}{\mathrm{~d} Z}=10^{-39} \frac{n_{10}^{2} \Sigma}{\mu^{*}\left(T_{6}\right) T_{6}^{2}}
$$

which will be needed in calculating the thermal conductive flux in the source.

\section{Energy Flow in the High Temperature Plasma}

Culhane et al. (1970) and Kahler (1971a, b) have already discussed energy loss processes in high temperature coronal flare plasmas. Their calculations are, ever, based on very simple models of the temperature structure - characterised by either a constant conductive flux (Kahler, 1971a) or a constant temperature gradient (Culhane et al., 1970) along some characteristic length. These representations are unsatisfactory since the temperature structures are not compatible with the observed $\mathrm{X}$-ray spectrum. Only by incorporating the X-ray spectral data into the temperature model is it possible to see how much the temperature gradient varies in the hot source, and, likewise, the conductive flux - i.e. to see the influence of energy sources on sinks.

The temperature profile derived as in Section 2 is by definition compatible with the $\mathrm{X}$-ray spectrum and provides the necessary basis for conduction calculations. As the most important example, this investigation will be carried out here only for the case of a power-law spectrum $F(\varepsilon) \sim \varepsilon^{-\gamma+1}$ as considered in Section 2, but rewritten here as

$$
F(\varepsilon)=(\gamma-1) I_{0}\left(\frac{\varepsilon}{\varepsilon_{0}}\right)^{-\gamma+1},
$$

where $I_{0}$ is the total flux $\left(\mathrm{cm}^{-2} \mathrm{~s}^{-1}\right)$ at the Earth of all photons of $\varepsilon \geqslant \varepsilon_{0}(\mathrm{keV})$ for some $\varepsilon_{0}$. In this case Equations (11), (14), (15), (16), (17) and (18) reduce (with $R=1 \mathrm{AU}$ ) to

if

$$
\begin{aligned}
& \mu^{*}\left(T_{6}\right)=\frac{0.36(\gamma-1)}{\Gamma(\gamma-1)} \frac{I_{0}}{\varepsilon_{0}^{-\gamma+1}}\left(\frac{T_{6}}{11.6}\right)^{-\gamma+1 / 2} \\
& \mathrm{~d} V\left(\mathrm{~cm}^{3}\right)=\frac{4.8 \times 10^{40}}{n_{10}^{2}} \frac{(\gamma-1)}{\Gamma(\gamma-1)} \frac{I_{0}}{\varepsilon_{0}^{-\gamma+1}}\left(\frac{T_{6}}{11.6}\right)^{-\gamma+5 / 2} \mathrm{~d} T_{6}
\end{aligned}
$$

while if

$$
\begin{aligned}
\gamma \neq 3.5, \quad \Delta V\left(T_{1}, T_{2}\right)= & \frac{5.6 \times 10^{41}}{n_{10}^{2}} \frac{(\gamma-1)}{\left(\gamma-\frac{7}{2}\right) \Gamma(\gamma-1)} \frac{I_{0}}{\varepsilon_{0}^{-\gamma+1}} \times \\
& \times\left[\left(\frac{T_{1}}{11.6}\right)^{-\gamma+7 / 2}-\left(\frac{T_{2}}{11.6}\right)^{-\gamma+7 / 2}\right]
\end{aligned}
$$

$$
\begin{aligned}
& \gamma=3.5, \quad \Delta V\left(T_{1}, T_{2}\right)=\frac{5.6 \times 10^{41}}{n_{10}^{2}} \frac{(\gamma-1)}{\Gamma(\gamma-1)} \frac{I_{0}}{\varepsilon_{0}^{-\gamma+1}} \log _{e}\left(\frac{T_{2}}{T_{1}}\right) \\
& \mathrm{d} Z=\frac{4.8 \times 10^{40}}{n_{10}^{2} \Sigma} \frac{(\gamma-1)}{\Gamma(\gamma-1)} \frac{I_{0}}{\varepsilon_{0}^{-\gamma+1}}\left(\frac{T_{6}}{11.6}\right)^{-\gamma+5 / 2} \mathrm{~d} T_{6}
\end{aligned}
$$


if

while if

$$
\begin{aligned}
\gamma \neq 3.5, \quad \Delta Z\left(T_{1}, T_{2}\right)= & \frac{5.6 \times 10^{41}}{n_{10}^{2} \Sigma} \cdot \frac{(\gamma-1)}{\left(\gamma-\frac{7}{2}\right) \Gamma(\gamma-1)} \frac{I_{0}}{\varepsilon_{0}^{-\gamma+1}} \times \\
& \times\left[\left(\frac{T_{1}}{11.6}\right)^{-\gamma+7 / 2}-\left(\frac{T_{2}}{11.6}\right)^{-\gamma+7 / 2}\right]
\end{aligned}
$$

and

$$
\gamma=3.5, \quad \Delta Z\left(T_{1}, T_{2}\right)=\frac{5.6 \times 10^{41}}{n_{10}^{2} \Sigma} \frac{(\gamma-1)}{\Gamma(\gamma-1)} \frac{I_{0}}{\varepsilon_{0}^{-\gamma+1}} \log _{e}\left(\frac{T_{2}}{T_{1}}\right)
$$

$$
\frac{\mathrm{d} T_{6}}{\mathrm{~d} Z}=2.1 \times 10^{-41} n_{10}^{2} \Sigma \frac{\Gamma(\gamma-1)}{(\gamma-1)} \frac{\varepsilon_{0}^{-\gamma+1}}{I_{0}}\left(\frac{T_{6}}{11.6}\right)^{\gamma-5 / 2}
$$

This can be solved to give the $Z$ dependence of the temperature structure, viz:

for

and for

$$
\gamma \neq \frac{7}{2}, \quad T_{6}=\left[\left(\frac{7}{2}-\gamma\right) \beta Z+10^{7 / 2-\gamma}\right]^{1 /(7 / 2-\gamma)}
$$

with $Z=0$

at $T_{6}=10$

$$
\gamma=\frac{7}{2}, \quad T_{6}=10 \exp (\beta Z)
$$

where

$$
\beta=\frac{2.1 \times 10^{-41}}{11.6^{\gamma-5 / 2}} n_{10}^{2} \Sigma \frac{\Gamma(\gamma-1)}{\gamma-1} \frac{\varepsilon_{0}^{-\gamma+1}}{I_{0}}
$$

Culhane et al. (1970) have demonstrated that, in the high temperature regime concerned here, energy transfer in the plasma is predominantly by the flow of thermal energy down the temperature gradient. They point out, however, that this flow can only be described as a thermal conduction process provided that the collision mean free path is shorter than the scale length of the temperature gradient. In an erratum to his paper (1971a), Kahler (1971b), using again a constant thermal flux model, found that this condition is not met at the high temperatures $\left(\sim 10^{8} \mathrm{~K}\right)$ required for hard X-ray emission. This can be better assessed by means of (25) which shows the scale distance for change of $T_{6}$ to be

$$
l \simeq \frac{\mathrm{d} Z}{\mathrm{~d} \log T}=\frac{5.6 \times 10^{41}}{n_{10}^{2} \Sigma} \frac{\gamma-1}{\Gamma(\gamma-1)} \frac{I_{0}}{\varepsilon_{0}^{-\gamma+1}}\left(\frac{T_{6}}{11.6}\right)^{-\gamma+7 / 2}
$$

while the collisional mean free path is (Billings, 1966)

so that

$$
L \simeq \frac{2.3 \times 10^{16}}{n} \times T_{6}=\frac{2.3 \times 10^{15}}{n_{10}} T_{6}^{2}
$$

$$
\alpha=\frac{L}{l}=5.3 \times 10^{-25} n_{10} \Sigma \frac{\Gamma(\gamma-1)}{\gamma-1} \frac{\varepsilon_{0}^{-\gamma+1}}{I_{0}}\left(\frac{T_{6}}{11.6}\right)^{-\gamma+11 / 2},
$$

where the regime $\alpha \ll 1$ corresponds to thermal conductive flow while with $\alpha \gg 1$ energy transfer will be essentially a matter of mass flow of hot material. 
Taking typical parameters from the large burst observed by Cline et al. (1968), viz. $\gamma \simeq 3.5$ and $I_{0} \simeq 300 \mathrm{~cm}^{-2} \mathrm{~s}^{-1}$ for $\varepsilon_{0}=80 \mathrm{keV}$ then

$$
\alpha=1.6 \times 10^{-30} n_{10} \Sigma\left(\frac{T_{6}}{11.6}\right)^{2} \text {. }
$$

The value of $n_{10}$ is somewhat uncertain but is probably in excess of $10^{10} \mathrm{~cm}^{3}$ (e.g. Neupert, 1969; Culhane et al., 1970) as determined by soft X-ray data. The area $\Sigma$ of the hard X-ray source is entirely unknown though Takakura et al. (1971) have given some evidence for its being very small - certainly smaller than the soft X-ray sources photographed by e.g. Vaiana et al. (1968) who put their characteristic size at around $10^{9} \mathrm{~cm}$. Thus the area $\Sigma$ is unlikely to exceed $10^{18} \mathrm{~cm}^{2}$ (cf. Culhane et al., 1970) so that

$$
\alpha \lesssim 1.6 \times 10^{-2}\left(\frac{T_{6}}{11.6}\right)^{2}
$$

Hence even at temperatures $\gtrsim 10^{8} \mathrm{~K}\left(T_{6} \gtrsim 100\right)$, $\alpha$ is only of order unity and conductive conditions might exist in the source. In view of the sensitivity of $\alpha$ on the various parameters in (26) and of the uncertainty in the values of these, however, it is appropriate to calculate the energy flow rates on both the thermal conduction approximation and on the mass flow basis.

Provided the conduction coefficient is not anomalously reduced by Plasma turbulence, then the conductive flux $F_{\text {cond }}$ will be as given in Spitzer (1962), viz.

$$
F_{\text {cond }} \simeq-7.6 \times 10^{14} T_{6}^{5 / 2} \frac{\mathrm{d} T_{6}}{\mathrm{~d} Z}
$$

which, with power-law gradient $(25)$ reduces to

$$
F_{\text {cond }}=-7.4 \times 10^{-24} n_{10}^{2} \Sigma \frac{\Gamma(\gamma-1)}{(\gamma-1)} \frac{\varepsilon_{0}^{-\gamma+1}}{I_{0}}\left(\frac{T_{6}}{11.6}\right)^{\gamma}
$$

which illustrates how the true temperature dependence of $F_{\text {cond }}$ differs here from the constant flux adopted by Kahler (1971a).

Following Kahler (1971b), the energy flux for a mass flow situation may be written as

$$
F_{\text {mass }} \simeq \frac{1}{4} n \bar{v} \bar{E} .
$$

If this mass flow followed the sudden creation of the high temperature situation, the density distribution $n(\mathbf{r})$ could initially be arbitrary since the configuration would be transient and constant pressure condition (25) would not apply. In fact the plasma distribution would readjust to near constant pressure on a time scale $\simeq l / \bar{v}$ which will always be short compared to typical durations of hard X-ray bursts (10-100 s). Subsequently the cooling of the hot plasma will approximate to the exchange of hot and cool material by nett flow at approximately constant pressure. Therefore during the sustained part of the burst, the mass energy flow can be written, using (25), as

$$
F_{\text {mass }} \simeq-0.35 n_{10} T_{6}^{1 / 2} \text {. }
$$


comparing (28) and (29), then

$$
\frac{F_{\text {cond }}}{F_{\text {mass }}} \simeq 2 \times 10^{-24} n_{10} \Sigma \frac{\Gamma(\gamma-1)}{\gamma-1} \frac{\varepsilon_{0}^{-\gamma+1}}{I_{0}}\left(\frac{T_{6}}{11.6}\right)^{\gamma-1 / 2}
$$

which, for the burst observed by Cline et al. (1968), gives

$$
\frac{F_{\text {cond }}}{F_{\text {mass }}} \simeq 10^{-3} \times\left(\frac{T_{6}}{11.6}\right)^{3}
$$

where $n_{10} \simeq 10^{10}$ and $\Sigma \simeq 10^{18}$ have been again assumed.

Equation (28) showed that, for this burst, the conductive cooling description breaks down for $T_{6} \geqslant 100$ (i.e. $T \geqslant 10^{8} \mathrm{~K}$ ). For these high temperatures, however, (33) shows that use of the conduction approximation description in fact overestimates the heat flow rate since $F_{\text {cond }}$ exceeds $F_{\text {mass }}$. (This situation differs from the results of Kahler, $1971 \mathrm{a}, \mathrm{b}$ due to the dependence of $F_{\text {cond }}$ on $\mathrm{d} T_{6} / \mathrm{d} Z$ which is correctly given as a function of $T_{6}$ by Equation (25) and not by Kahler's empirical model.) Thus to investigate the feasibility of sustaining a multitemperature flare plasma to $T \geqslant 10^{8}$ it will be conservative to adopt the conductive cooling approximation throughout, as will be done from this point on.

The actual effect of the conductive flow on the temperature is given by considering the characteristic time $\tau_{c}=\mathscr{E} / \nabla \cdot \mathbf{F}$ where $\mathscr{E}$ is the internal energy $\left(\mathrm{erg} \mathrm{cm}^{-3}\right)$ and $\mathbf{F}$ is the vector thermal energy flux. Differentiating (30) in the present one-dimensional case, and using (25), gives

$$
\begin{aligned}
\nabla \cdot \mathbf{F} & =\frac{\mathrm{d} F_{\text {cond }}}{\mathrm{d} Z}=\frac{\mathrm{d} F_{\text {cond }}}{\mathrm{d} T_{6}} \times \frac{\mathrm{d} T_{6}}{\mathrm{~d} Z}= \\
& =-1.3 \times 10^{-65} \gamma\left[n_{10}^{2} \Sigma \frac{\Gamma(\gamma-1)}{(\gamma-1)} \frac{\varepsilon_{0}^{-\gamma+1}}{I_{0}}\right]^{2}\left(\frac{T_{6}}{11.6}\right)^{2 \gamma-7 / 2}
\end{aligned}
$$

while

$$
\mathscr{E}=3 n k T_{6} \times 10^{6}=3 \times 10^{7} n_{10} k=4.2 \times 10^{-9} n_{10},
$$

i.e. the energy density is constant through the source (due to constant pressure conditions).

Combining (34) and (35), then

$$
\tau_{c} \simeq-\frac{3.1 \times 10^{56}}{n_{10}^{3} \Sigma^{2}} \frac{(\gamma-1)^{2}}{\gamma(\Gamma(\gamma-1))^{2}}\left(\frac{I_{0}}{\varepsilon_{0}^{-\gamma+1}}\right)^{2}\left(\frac{11.6}{T_{6}}\right)^{2 \gamma-7 / 2} .
$$

This is the key result required and contains all the information needed about $\tau_{c}$ as a function of the observed flare parameters. If differs from the corresponding result from Kahler (1971a, b) and also from Culhane et al. (1970) in a number of respects, of which two are crucial.

Firstly, the negative sign of $\tau_{c}$ indicates that, in order to fit an X-ray power-law spectrum, the plasma is (locally) not cooled by conduction at all but rather heated. This is a consequence of the concavity of the function $F_{\text {cond }}(Z)$ (as against the convex 
form arising in previous empirical models) similar to the situation obtaining at the base of the quiet chromosphere transition region (cf. for example Kuperus, 1969). At high enough temperatures this state of affairs must break down, the temperature gradient inflecting into the region of maximum temperature (and appearing as a break in the hard X-ray spectrum such as is observed in fact - e.g. Frost, 1969) from which the energy flow required by (36) would ultimately emanate. Thus, though $\tau_{c}$ is in fact a heating rather than a cooling time, its significance in the present context is unchanged since it still represents the characteristic time for conductive redistribution of thermal energy in the source, and hence the time scale on which temperature structure (25), could survive in the absence of sustained energy supply (a factor considered in the next section).

The second important aspect of (36) is that it shows $\tau_{c}$ to be very sensitive to the adopted values of $n_{10}$ and $\Sigma$ as well as to the local temperature $T_{6}$ (depending on index $\gamma)$. Any substantial uncertainty in assessing either $n_{10}$ or $\Sigma$ will therefore have a critical effect on the feasibility of the multi-thermal model for hard X-ray emission. Detailed results for the large event of Cline et al. (1968) are given in the next section but, bearing in mind the uncertainties involved, a tentative value of $\tau_{c}$ can be assigned for this event using the values for $n_{10}$ and $\Sigma$ mentioned above. These give

$$
\tau_{c}(\mathrm{~s}) \lesssim 1.5 \times 10^{5} \times\left(\frac{11.6}{T_{6}}\right)^{3.5}
$$

so that even at $T_{6}=116$ (i.e. $1.16 \times 10^{8} \mathrm{~K}$ ) the time $\tau_{c} \simeq 50 \mathrm{~s}$ which is within a factor of 2 of the observed decay time of this burst at $80 \mathrm{keV}$. Thus, with these parameters, the conductive temperature redistribution could actually be identified with the burst decay, contrary to the results and conclusions of Kahler (1971a, b). However, since $\tau_{c}$ is so sensitive to $n_{10}$ and $\Sigma$ (e.g. increasing $n_{10}$ threefold decreases $\tau_{c}$ from 50 to $2 \mathrm{~s}$ ), this identification is probably fortuitous. As discussed in the following section, further considerations indicate that $\tau_{c}$ is indeed probably smaller than the observed burst decay time. This would merely mean, however, that a continuing energy input was required through the burst duration and the above expression for $\tau_{c}$ allows evaluation of the required input which is shown below to be feasible.

\section{Detailed Results for a Large Event}

Some of the analytical results of the previous sections may be clarified by their representation in numerical form for a particular event, which will be chosen once again as that observed by Cline et al. (1968), for which, as already stated, $I_{0}=300 \mathrm{~cm}^{-2}$ $\mathrm{s}^{-1}$ for $\varepsilon_{0}=80 \mathrm{keV}$ and $\gamma=3.5$. Considering first the differential emission measure $\mu^{*}\left(T_{6}\right)$, this may be tabulated using (20) for a variety of temperatures, (see also Figure 1), see below.

These figures may be compared with those suggested by Chubb (1970), viz. an emission measure of around $10^{46} \mathrm{~cm}^{-3}$ for a $10^{8} \mathrm{~K}$ plasma. The corresponding figure 
here is

$$
\mu_{\mathrm{tot}}\left(T>10^{8}\right)=10^{41} \int_{100}^{\infty} \mu^{*}\left(T_{6}\right) \mathrm{d} T_{6}
$$

which by $(20) \simeq 10^{47} \mathrm{~cm}^{-3}$ - a somewhat greater value than Chubb's but in adequate agreement when it is noted that the event recorded by Cline et al. is one of the largest and hardest ever recorded.

\begin{tabular}{lllllll}
$T_{6}$ & 10 & 20 & 40 & 100 & 200 & 400 \\
\hline$T(\mathrm{~K})$ & $10^{7}$ & $2 \times 10^{7}$ & $4 \times 10^{7}$ & $10^{8}$ & $2 \times 10^{8}$ & $4 \times 10^{8}$ \\
$\mu^{*}\left(10^{41} \mathrm{~cm}^{-3} \operatorname{per~} 10^{6} \mathrm{~K}\right)$ & $2 \times 10^{7}$ & $2.5 \times 10^{6}$ & $3 \times 10^{5}$ & $2 \times 10^{4}$ & $2.5 \times 10^{3}$ & $3 \times 10^{2}$ \\
$\mu(T)\left(\mathrm{cm}^{-3} \operatorname{per~} 10^{6} \mathrm{~K}\right)$ & $2 \times 10^{48}$ & $2.5 \times 10^{47}$ & $3 \times 10^{46}$ & $2 \times 10^{45}$ & $2.5 \times 10^{44}$ & $3 \times 10^{43}$ \\
\hline
\end{tabular}

Equation (22) provides the volume of the emitting plasma when evaluated between the probable temperature $T_{1}\left(\simeq 4 \times 10^{7} \mathrm{~K}\right)$ at which the region becomes isothermal and an upper limit $T_{2}$ of around $10^{8} \mathrm{~K}$. Then

$$
\Delta V \lesssim 10^{29} \mathrm{~cm}^{3}
$$

for $n_{10} \gtrsim 10^{10} \mathrm{~cm}^{3}$. (This also follows approximately from $\mu_{\mathrm{tot}}=10^{47} \mathrm{~cm}^{-3}$ and a value of $10^{9} \mathrm{~cm}^{-3}$ for $n$ at $T=10^{8} \mathrm{~K}$.) This volume is rather large since for a source filament cross-section $\Sigma \simeq 10^{18} \mathrm{~cm}^{2}$, the corresponding length $\Delta Z \simeq 10^{11} \mathrm{~cm}$ exceeds a solar radius. Though no high resolution hard $\mathrm{X}$-ray data exist to test the existence of such extensive sources, a single source of this size seems improbable. The problem may be eluded however, if the source consists of two filaments (c.f. Culhane et al., 1970) like the soft X-ray flare (Vaiana et al., 1968) each of $\Delta Z \simeq 5 \times 10^{10} \mathrm{~cm}$ (i.e. a solar radius) in the form of arched coronal plasma traps. Kahler(1971a) expresses the view that such an extended source is unlikely since the energy source would have to be very extended also. This is not so since, with the correct expression for $T(Z)$, conduction has been shown here to supply energy along the filament from a source at the high temperature end where the basic flare energy release may occur-i.e. for example, the required thermal X-ray source temperature structure could be sustained by conduction from an extremely hot coronal region heated in a plasma turbulent neutral sheet configuration.

However, such lengths are not in any case essential to the model considered here since they can be reduced by increase of $n_{10}$ or of $\Sigma$, though this at the expense of a decreased decay time $\tau_{c}$ (Equation (36)) so that energy would have to be continuously supplied to sustain the temperature structure required by the burst. A twofold increase of both $n_{10}$ and $\Sigma$ would reduce $\Delta V$ by a factor of 4 and $\Delta Z$ by a factor of 8 (Equations (22) and (24)) but would reduce the time $\tau_{c}$ for the event of Cline et al. (1968) to about $2 \mathrm{~s}$ (Equation (36)). The total input energy flux required to sustain the burst thermally would then be the value of $F_{\text {cond }}$ (Equation (30)) near the maximum temperature $\left(T_{6}^{\max }\right)$ 
present - i.e. at the inflection point in $\mathrm{d} T / \mathrm{d} Z$, viz.

$$
F_{\text {in }}=F_{\text {cond }} \simeq 1.6 \times 10^{8}\left(\frac{T_{6}}{11.6}\right)^{3.5} \text {. }
$$

At $T=10^{8} \mathrm{~K}$, this gives $F_{\text {in }} \simeq 3 \times 10^{11} \mathrm{erg} \mathrm{cm}^{-2} \mathrm{~s}^{-1}$ at $10^{8} \mathrm{~K}$ which is a large energy flux but over the filament area of $2 \times 10^{18} \mathrm{~cm}^{2}$ for the $100 \mathrm{~s}$ necessary, the total energy input required is about $6 \times 10^{31} \mathrm{erg}$ which is easily available in an event this large. This particular burst, was, however, so hard that the temperature needed to produce it entirely thermally would be nearer $10^{9} \mathrm{~K}$ than $10^{8} \mathrm{~K}$ (c.f. Figure 1) whereas Equation (37) shows that a reasonable maximum for $F_{\text {in }}$ restricts $T$ to $\lesssim 2 \times 10^{8} \mathrm{~K}$. Within the terms of the preceding analysis, this would require that the thermal component of hard X-ray bursts give way continuously to non-thermal emission at the highest photon energies. Such a smooth transition between the two components is entirely reasonable, especially if the hot plasma and the high energy tail of particle streams are generated in one and the same plasma turbulent region. (Potential methods of observing this transition energy are discussed briefly in Section 6, while its importance as regards the entire question of flare energetics has already been emphasized in Section 1.) On the other hand, the above results are based purely on the assumption of normal thermal conductivity. It is entirely conceivable that the conductivity throughout the source may be reduced by the presence of plasma turbulence and by factors of up to $10^{4}$. This would of course totally eliminate any objections to the thermal model based on the argument of rapid conductive cooling, even up to the hardest photons observed by Cline et al. (1969).

Fuller consideration of these latter features of the problem must, however, incorporate more specific models of the X-ray emitting plasma and of its energy source, and is therefore postponed till a future paper. The aim here has been to emphasize that the possibility of high temperature plasmas as the source of hard $\mathrm{X}$-ray flares cannot be excluded on energy grounds within the limits of presently available observations. The main observations needed to resolve the issue are some limits on the dimensions of the hard X-ray flare, presumably from occultation or collimator experiments. Lacking these, there are two other observational data to which appeal may be made. viz. the polarization and the time variations of hard X-ray bursts. The relationship of these to the source model described above is discussed briefly below.

\section{Polarization and Temporal Fine Structure in Hard X-Ray Flares}

It has been frequently supposed (e.g. Brown, 1971; Tindo et al., 1970, 1973) that the observation of substantial polarization (up to around $40 \%$ ) in flare hard X-rays (Tindo et al., 1970, 1972, 1973) and also perhaps of directivity (Ohki, 1969; Pintér, 1969; Drake, 1971) is indicative of non-thermal processes, and particularly of electron streams. Recently, however, Tomblin (1972) drew attention to the contribution in observed soft X-ray fluxes of source photons reflected by the photospheric albedo. 
Santangelo et al. (1973) have shown this contribution to be even more important at high photon energies, being a large proportion of the total observed flux in the hard $\mathrm{X}$-ray range. In common with all such scattered radiation, as pointed out by Santangelo et al. (1973), these albedo X-rays will be partially linearly polarized, thus modifying previous calculations of the polarization and directivity of non-thermal hard X-rays (e.g. Haug, 1972; Brown, 1972b). More important in the present context, however, is that photospheric scattering will introduce polarization and anisotropy into the X-rays observed from a thermal source above the photosphere, this source being itself unpolarised and isotropic. Thus the presence of polarization and anisotropy in hard X-ray flares does not after all preclude a multi-temperature thermal source. There is therefore an urgent need for quantitative work on the expected extent of these effects. Beigman and Vainshtein (1974) have calculated about $2 \%$ polarization introduced by albedo photons in the case of thermal soft $\mathrm{X}$-rays but do not investigate the hard X-ray case. Considering the much greater albedo contribution at hard energies, however, it seems likely that the polarization introduced will also be considerable, perhaps comparable with that observed.

As regards the plane of albedo polarization, it would be expected that the maximum intensity occurs normal to the scattering plane -i.e. approximately normal to the solar radius through the source region (Santangelo et al., 1973), unless the scattering is in some way anomalous as in the case of lunar polarization near full phase (Dollfus, 1966). This prediction does not in fact agree with the sparse observational data available (Tindo et al., 1972) which indicate that for a few bursts the emission is partially polarized approximately along the solar radius through the flare - a result which may be interpreted in terms of vertically streaming electrons (Tindo et al., 1972; Brown, 1972b) though this interpretation may be in doubt (Brown et al., 1974). With the reservations expressed at the end of Section 5, it seems likely that the X-ray emission mechanism does become non-thermal at high enough energies but the energy at which the transition occurs is the all important question and may vary from flare to flare. If a 'radial' polarization plane is the rule rather than the exception for the nonthermal component then observations of the variation of the polarization plane with photon energy may provide the key to determining where thermal emission gives way to non-thermal. For at low enough energies, the plane must go over to the tangential case characteristic of scattering polarization, the transition occurring where thermal processes become dominant. A plea is therefore in order for theoretical work on albedo hard X-ray polarization and for observations of the energy dependence of the polarization.

Turning finally to the time dependence of the observed hard X-ray flux from flares, rapid time variations $(\simeq$ seconds), well correlated with (non-thermal) microwave emission, are typical features (Frost, 1969; Parks and Winckler, 1969, 1971) which Kahler (1971a) for example, regards as uncharacteristic of thermal emission. Though the microwave burst (gyro-synchrotron) mechanism certainly involves non-thermal electrons (Takakura, 1967), it does depend on the flare field strength $H$ in the emitting region. This field may vary rapidly with time due to magnetohydrodynamic waves 
with concomitant variations in the density of the frozen-in ambient plasma (e.g. Parks and Winckler, 1969, 1971; Wilson, 1972; Brown, 1973c). Such a situation could produce the observed time structure of microwave emission from trapped high energy (non-thermal) electrons and closely correlated variations in the ambient plasma density $n$ and hence in any thermal X-ray emission arising from it (Equation (1)). Though this proposal is certainly subject to detailed investigation, it should serve to show that temporal fine structure in hard X-ray flares may not be taken out of hand as precluding a multi-temperature thermal emission mechanism. (The definite presence of nonthermal electrons required for microwave emission is not contrary to this view since the electron numbers involved are only $10^{-3}$ of those needed for the X-ray bremsstrahlung.) The factor which would ultimately limit the range of validity of such a description is that, at high enough temperatures, the electron mean free path (and hence the Maxwell relaxation time) becomes too great (Equation (26)) for description of the electron distribution as thermal at all within the observed rapid time structures (Kahler, 1971b). Above this limit the usual distinction between ambient and non-thermal electrons will be come meaningless. However, as shown by the equation preceding (26), for a density $n_{10} \simeq 10^{10} \mathrm{~cm}^{-3}$, and time structure of a few seconds this limitation does not set in until $T \gtrsim 5 \times 10^{8} \mathrm{~K}$ - i.e. well into the regime normally regarded as non-thermal. Furthermore, the reduction of conductivity by turbulence, would permit much higher values of $n_{10}$ in the model described here and consequent Maxwellian relaxation times much shorter than any observed hard X-ray fine structure. Hence it must be concluded that the important question of the relative importance of non-thermal and high temperature electrons in fiare hard X-ray bursts remains open to fuller observational and theoretical investigation.

\section{Acknowledgements}

My thanks are due to Dr Steve Kahler for constructive criticisms of the preprint of this paper, and to the IAU, the Australian Academy of Sciences, and to Glasgow University whose support enabled me to attend this Symposium.

\section{References}

Abramowitz, M. and Segun, I. A.: 1965, Handbook of Mathematical Functions, Dover.

Batestone, R. M., Evans, K., Parkinson, J. H., and Pounds, K. A.: 1970, Solar Phys. 13, 389.

Beigman, I. L. and Vainshtein, L. A.: 1974, Astron. J. (USSR), in press.

Billings, D. E.: 1966, A Guide to the Solar Corona, Academic Press, N.Y.

Brown, J. C.: 1971, Solar Phys. 18, 489.

Brown, J. C.: 1972a, Solar Phys. 25, 158.

Brown, J. C.: 1972b, Solar Phys. 26, 441.

Brown, J. C.: 1973a, Solar Phys. 28, 151.

Brown, J. C.: 1973b, Solar Phys. 31, 143.

Brown, J. C.: 1973c, Solar Phys. 32, 227.

Brown, J. C., McClymont, A. N., and McLean, I. S.: 1974, Nature 247, 448.

Chambe, G.: 1971, Astron. Astrophys. 12, 210.

Chubb, T. A., Kreplin, R. W., and Friedman, H.: 1966, J. Geophys. Res. 71, 3611. 
Chubb, T. A.: 1970, in E. R. Dyer (ed.), Proc. Leningrad Symp. Solar Terrestrial Phys., Vol. 1, D. Reidel Publ. Co., Dordrecht, Holland.

Chupp, E. L., Forrest, D. J., Higbie, P. R., Suri, A. N., C., and Dunphy, P. P.: 1973, Nature $241,333$.

Cline, T. L., Holt, S. S., and Hones, E. W.: 1968, J. Geophys. Res. 73, 434.

Culhane, J. L.: 1969, Monthly Notices Roy. Astron. Soc. 144, 375.

Culhane, J. L., Vesecky, J. F., and Phillips, K. J. H.: 1970, Solar Phys. 15, 394.

Dollfus, A.: 1966, in Hess, Menzel, and O'Keefe (eds.), The Nature of the Lunar Surface, John Hopkins, Press.

Drake, J. F.: 1971, Solar Phys. 16, 152.

Elwert, G. and Haug, E. : 1971, Solar Phys. 20, 413.

Felten, J. E. and Rees, M. J.: 1972, Astron. Astrophys. 17, 226.

Fichtel, C. E. and McDonald, F. B.: 1967, Ann. Rev. Astron. Astrophys. 5, 351.

Frost, K. J.: 1969, Astrophys. J. 158, L.159.

Greisen, E.: 1971, Physics of Cosmic X-Ray, $\gamma$-Ray and Particle Sources, Gordon and Breach.

Herring, J. R. H. and Craig, I. J. D. : 1973, Solar Phys. 28, 169.

Haug, E.: 1972, Solar Phys. 25, 425.

Illarionov, A. F. and Sunyaev, R. A.: 1973, Astrophys. Space Sci. 19, 61.

De Jager, C.: 1967, Solar Phys. 2, 327.

Kahler, S. W.: 1971a, Astrophys. J. 164, 365.

Kahler, S. W.: 1971b, Astrophys. J. 168, 319.

Kane, S. R. and Anderson, K. A. : 1970, Astrophys. J. 162, 1003.

Kane, S. R. and Donnelly, J. E.: 1971, Astrophys. J. 164, 151.

Kuperus, M.: 1969, Space Sci. Rev. 9, 713.

Milkey, R. W.: 1971, Solar Phys. 16, 465.

Neupert, W. M.: 1968, Astrophys. J. 153, L.59.

Neupert, W. M.: 1969, Ann. Rev. Astron. Astrophys. 7, 121.

Ohki, K.: 1969, Solar Phys. 7, 260.

Parks, G. K. and Winckler, J. R.: 1969, Astrophys. J. 155, L117.

Parks, G. K. and Winckler, J. R.: 1971, Solar Phys. 16, 186.

Pintér, S.: 1969, Solar Phys. 8, 142.

Santangelo, N., Horstman, H., and Horstman-Moretti, E.: 1973, Solar Phys. 29, 143.

Sneddon, I. N.: 1972, The Use of Integral Transforms, McGraw Hill.

Spitzer, L.: 1962, Physics of Fully Ionised Gases, Interscience, New York.

Takakura, T.: 1967, Solar Phys. 1, 304.

Takakura, T., Ohki, K., Shibuya, N., Fujii, M., Matsuoka, M., Miyamoto, S., Nishimura, J., Oda, M., Ogawara, Y., and Ota, S.: 1971, Solar Phys. 16, 454.

Tindo, I. P., Ivanov, V. D., Mandelstam, S. L., and Shuryghin, A. I. : 1970, Solar Phys. 14, 204.

Tindo, I. P., Ivanov, V. D., Mandelstam, S. L., and Shuryghin, A. I. : 1972, Solar Phys. $27,426$.

Tindo, I. P., Mandelstam, S. L., and Shuryghin, A. H.: 1973, Solar Phys. 32, 469.

Tomblin, F. F.: 1972, Astrophys. J. 171, 377.

Vaiana, G. S., Reidy, W. P., Zehnpfennig, T., Van Speybröck, L., and Giacconi, R. : 1968, Science $161,564$. Wilson, P. R.: 1972, Solar Phys. 22, 434.

\section{DISCUSSION}

I suggest that if such large amounts of very hot plasma are assumed to account for hard, fast X-ray bursts then it is difficult to account for the following flare observations:

(1) Observed weakness of $L \alpha$ radiation from Fe XXVI.

(2) Small $\left(<1^{\prime}\right)$ hard X-ray source size measured by Oda et al.

(3) Close time profile correlation between $\mu$-wave, EUV and hard X-ray bursts.

Brown: The $\mathrm{L} \alpha$ of iron at $1.8 \AA$ would be gone at those temperatures.

Acton: But it should appear during the cooling phase.

Smith (to Acton): It is completely possible to heat plasma very rapidly by dumping the energy into wave modes which are heavily damped such as ion-acoustic waves.

Kane: I think that the objection No. 3 mentioned by you, viz. the difficulty in explaining the rapid time variations, should be taken seriously. To illustrate how difficult this problem can get, I would like to show 
a slide. This slide shows at the top $10-580 \mathrm{MHz}$ spectral radio data obtained by Dr Maxwell and at the bottom OGO- $5 \mathrm{X}$-ray data above $20 \mathrm{keV}$ energy. Corresponding to the 5 groups of type III bursts in the radio data, there are 5 peaks in the X-ray data. This strongly suggests that, like the type III radio emission, the time variation in the $\mathrm{X}$-ray emission may have a time constant of $\sim 0.1 \mathrm{~s}$ which is very difficult to explain by a thermal model of the $\mathrm{X}$-ray source.

Meyer: Your assumed conditions appear pretty far from pressure equilibrium with the surroundings.

Sturrock: In connection with the thick/thin X-ray target controversey, Petrosian has recently analyzed the problem of a vertical electron beam and finds the polar diagram concentrated downward and a strong spectral variation with direction. He concludes that this makes the deduction of the electron spectrum from the X-ray spectrum unreliable.

Brown: I have not seen this paper but my own analysis of that problem showed that the X-ray spectral index varied by less than 0.6 with direction, which is not very significant. On the other hand, the intensity can vary by about 5 . It may be that Petrosian neglected scattering and so over-estimated the beam collimation. 